\title{
PERFORMNACE ANALYSIS OF OPPORTUNISTIC ROUTING PROTOCOLS FOR MANETS
}

\author{
Dr. K. Pushpalatha* \\ Associate Professor, Department of Information Technology, Coimbatore Institute of \\ Engineering and Technology, Coimbatore-641109 \\ Dr.K.Poorananapriya \\ Professor, Department of ECE, Vidyaa vikas College of Engineering and Technology, \\ Thiruchengodu-637214 \\ Mrs.J.Angelin Sheela \\ Assistant Professor,Department of Information Technology \\ Coimbatore Institute of Engineering and Technology, Coimbatore-641109
}

\begin{abstract}
The advancements in the wireless networks have increased the utilization of wireless devices in emergency situations such as disaster relief and rescue operations, hurricane affected regions and underground mines and so on. Traditional routing protocols of Mobile Adhoc networks are not appropriate for such scenarios because it requires constant path for its communication. Hence, in order to surmount the restriction of traditional routing protocols, and to increase the network performance in emergency situations, the opportunistic routing paradigm has been suggested in recent research works. The purpose of Opportunistic Routing protocols is to increase the reliability of delivering data packets to their destination by using the broadcast nature of the wireless medium. In this paper, we provide a comprehensive study of the existing literature and performance analysis of opportunistic routing protocols.
\end{abstract}

Keywords: Mobile Adhoc Networks, Opportunistic routing, Wireless technology, Routing , Reliability.

\section{Introduction}

In recent years, Mobile Ad hoc Networks (MANETs) has turn the important region of research because of the challenges confronted by its routing protocols. MANET is a group of nodes forming a temporary network without the aid of any predefined infrastructure. These wireless nodes are able to communicate with its neighbours, even in the lack of infrastructure [1]. 


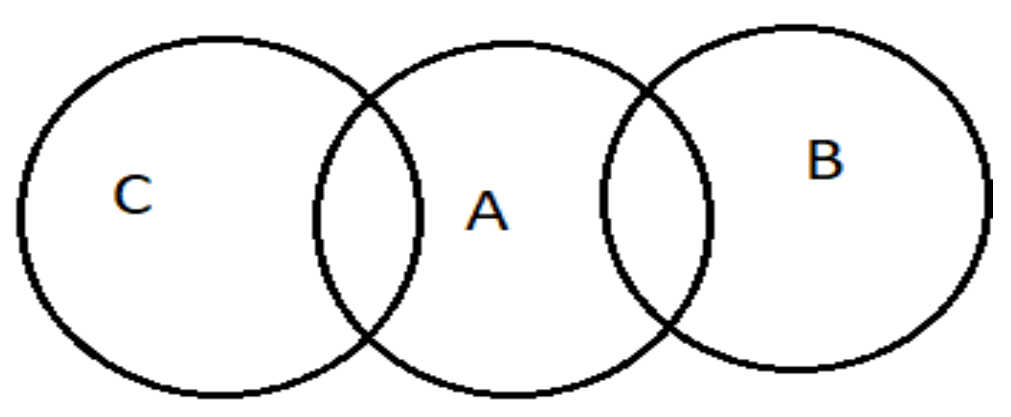

Fig 1. Mobile Ad hoc Network

The nodes that are present in each other's transmission range can directly communicate among themselves. If not, it requires the assistance of other nodes for forwarding the packets. The Fig. 1 shows the Mobile Ad hoc Network. The nodes A and B and the nodes A and C are directly communicating with each other, whereas the nodes $\mathrm{C}$ requires the assistance of node A for communicating with the node $\mathrm{B}$ as node $\mathrm{C}$ is not in the direct transmission range of $\mathrm{B}$. These types of networks can be implemented anywhere, any place at any time. At any time, a node can come in and go to the network transmission range. Hence the topology of the network changes from time to time.

The performance of the network is determined by its underlying routing protocol. Over these years a number of protocols have been suggested for solving the routing problem in wireless networks.A clump of research work has been taken out in the past to improve the performance analysis of traditional routing protocols. Based on the analysis, the protocols like DSR [2], ZRP [3] and AODV [4] faces difficulties in handling dynamic topology and unpredictable wireless medium. Recent studies show these traditional routing protocols encounters numerous difficulties in handling with unpredictable wireless medium and random moving nodes. Most of these protocols rely on choosing the fixed path before communication takes place. But it is very hard to hold a defined path or a fixed topology for forwarding the packets in high mobility environments. Once the communication path breaks, the data would be lost which leads to disruption in the communication [5].

To conclude, traditional routing protocols are not suitable as it requires constant path for its communication. The route is established before the communication takes place. But it is very difficult to predict a constant path in such situation. Alternatively Opportunistic Routing $(\mathrm{OR})$ requires great attention nowadays as it utilizes the advantage of broadcasting nature of the wireless medium for its communication [6].

In this OR routing, the relay node is determined by the potential ability of nodes from its neighbourhood. Instead of selecting the predetermined path, OR transmits a data packet so that it is received by multiple nodes in its neighbourhood which later form the candidate relay set. Further, the best relay node is selected from the candidate relay set for forwarding the 
packets. The same process is repeated until the data packet reaches the destinations. So the packet retransmissions are greatly reduced in OR routing, which leads to increase in the performance of the network. This property has encouraged to use of OR in wide range of emergency applications. By considering the increasing importance of OR in today's communications, we provide the comprehensive study of various OR techniques in recent technical literature.

The rest of this paper is organized as follows. Section 2 presents components and classification of OR routing techniques. Section 3 discusses the various OR protocols in the recent technical literature. Section 4 presents the summary and future research directions. Finally section 5 concludes the paper.

\section{CLASSIFICATION OF OR ROUTING PROTOCOLS}

As discussed, In OR routing, the node broadcasts a data packets to its neighbours instead of forwarding it to preselected single node. These candidate nodes are prioritized to specific metric like the position of the node, trust and energy etc.,Based on these metrics, the best relay node is selected from the candidate node set to forward the packet. This process is repeated until the packet reaches the destination. Thus the operation of OR consists of four steps.

1. Data broadcasting.

2. Candidate node Set construction.

3. Relay node selection.

4. Data forwarding.

In OR routing, each node sends a packet to multiple nodes simultaneously. Hence, if any one of the node fails, any other node that received the packet can pass it on. The candidate node set is built based on receiving data packets and are prioritized based on some specific metric.To avoid duplicate transmissions, the highest priority node from the candidate relay set is selected as a relay node for forwarding the packets. The priority metric choice is depends on routing protocol objectives and requirements. In military like applications, security is more important and Emergency like applications, the device location is an important criteria for saving many people lives. Therefore the relay node is selected either based on security or with the location which is closest to the destination node[7]. The following Fig 2 shows the classification of OR routing technique based on relay node selection.

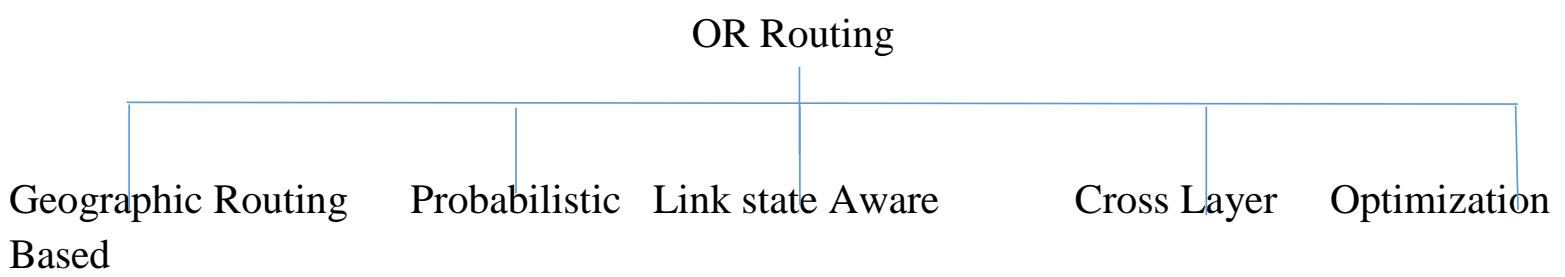

Fig 2. Classification of OR routing 


\subsection{GEOGRAPHIC OPPORTUNSTIC ROUTING}

This type of routing is based on the position information of the nodes. It overcomes the lack of infrastructure in mobile ad hoc network and adapts to frequent updating in network topologies.

Fussler et al. [8]haveproposed a geographic opportunistic routing protocol,Contention Based Forwarding $(\mathrm{CBF})$, in which the node which is nearest to the destination among all the neighbours is selected as a relay node for forwarding the data packet. This relay node selection is performed opportunistically via cooperation among multiple nodes in its neighbourhood. In this routing, the node who wishes to send the packet broadcasts an RTF (Request to Forward) packet to all its neighbours, and its neighbour nodes compete with each other to reply with a CTF (Clear to Forward) packet. The CTF transmission is determined based on distance and timer, thus that the receiver with the minimum distance replies first. Once the process is completed, the sender transmits the packet to this selected relay node. The process is repeated until the packet reaches the destinations. It does not require of any exchange of topology information for sending the packets as for the situation in traditional routing. However it consists of delay because of RTF and CTF control messages exchanges and the waiting time for the CTF reply.

Zeng et al. have proposed Geographic Opportunistic Routing (GOR) [9], an improvedversion of the CBF protocol. GOR protocol is designed to defeatthe limitations of $\mathrm{CBF}$ protocol. In this protocol, timer based coordination is used for reducing the overhead.The candidate relay nodes are sorted according to EOT metric. It considers both distance delay cost incurred by the coordination process. Thus the node with the highest priority will get a chance to forward a packet.Based on the experimental results, it evidences that the throughput is increased by GOR compared to CBF. It also relies on packet overhearing which prevents lower priority nodes transmitting the same packet at some extent. But it does not prevent the duplicate transmissions completely.

Yang et al [10] have proposed Position based Opportunistic Routing (POR), which is deployed to give robust packet delivery in critical Wireless Ad hoc networks where malicious nodes expeditiously drop packets. The position information is utilized in the routing module for supporting the mobility in multi-hop wireless networks.

In addition to handling portable wireless networks, POR supports multicast transmissi ons as well.It does not just deal with mobile wireless networks, but it supports multicast transmissions also. However, these multicast transmissions may introduce packet redundancy and randomness, thereby arriving at the forwarding of packets robust to node failure or attacks. Based on the experimental outcomes, POR allows more than $90 \%$ of the packets to be delivered in a moderate MANET with all nodes maliciously dropping the data packets with the probability of 50\%. However, this efficiency is reached at the detriment of increased duplicate transmissions and buffer occupancy. All of the aforementioned geographic opportunistic routing protocols use the Unit Disk Graph Model that characterizes the propagation using 
coverage circles. It may not be the best model for geographic routing, especially in high mobility and traffic wireless networks as it does not take quality of service into consideration.

\subsection{LINK STATE AWARE OPPORTUNISTIC ROUTING}

Based onprobabilities in the delivery of packets inwireless networks, the Link state aware routing protocol wasproposed by Biswas et al. that deals with the ExOR [11], which is the earlierlink state aware opportunisticroutingframework.The routing and MAC (Medium Access Control) operations are integrated into this protocol.Initially, the sender sendsa batch of 10 to 100 packets with a set of nodes that could possibly send the packets to suitable destinations. In the forwarding relay node set, each neighbour waits for their turn for further transmitting the received packets. The author had proposed a TDMA based MAC scheduling scheme that enforces the relay priority in the forwarding set, so that the candidate relay on forwarding the packets only if all higher priority candidates fails. This priority is quantified using the Expected Transmission Count, ETX metric that addresses the expected number of transmissions that are required to route a packet to the destination. These ETXvaluesaredetermined based on the information of the complete set of inter-node loss rates. The EXOR protocol increases the throughput in terms of link-state awareness property by a factor of two to four compared to traditional single path routing. A serious shortcomingsare discussedrelating to the forwarders coordination. The coordination method creates duplicate transmissions, particularly when the candidate forwarders are connected with low-quality links.

Hsu et al.[12] proposedECONOMY, a duplicate-free ETXbasedopportunisticroutingprotocol. To overcome the problem of duplicate transmissions, this protocol filters out the candidate relays that are not able to hear one another that is fully connectedwith CRSs. The ECONOMY uses token passing, in which only one token holder is allowed to forward packets. The simulation findingshave shownthat this protocol uses only a fewer amount of transmissions for transmitting a packet to the destination compared to ExOR. The performance rating in Economy is up to $100 \%$ which is compared to traditional routing in terms of throughput.This efficiency increase illustrates, this protocol induces a significant overhead that is prohibitive in low power Wireless Sensor Networks.

In reference to [13], Chachulski et al. introduced a MAC-Independent Opportunistic Routing and Encoding Protocol (MORE), which uses both opportunistic routing and intra-flow network coding that increases end-to-end throughput of the network. The MORE is also the basic opportunistic routing scenario to adopt network coding as the coordination method. Also, the MORE divides initial data packets into batches, each with $\mathrm{K}$ packets. The source node continuously transmits inrandom linear combination of $\mathrm{K}$ original data packets in the same batch. The receiving node which is closest to the destination in the ETX is subsequently chosen as the next forwarder. The forwarder only stores the packets that are linearly independent of the previously received packets in the same batch in order to avoidpacket duplication. Upon receiving $\mathrm{K}$ linearly independent packets, the destinationnoderestorestheoriginaldata packets received and later issues an Acknowledgment (ACK) after the reception of the packets thus allowing it to forward on to the next batch. The 
results shows that MORE achieves greater throughput than ExOR and traditional routing. In the absence of hop-by-hop acknowledgements in MORE, relays do not knowwhen to stop transmitting, which results in triggering of redundant packet transmission.

Cumulative coded acknowledgements(CCACK) [14], a more efficient opportunistic routing protocol based on network coding, also includes a new cumulative model that provides acknowledgement system for secure packet transmission. The system also enables nodes to recognize network coded traffic with virtually zero overhead to the upstream nodes. CCACK utilizes a network based acknowledgement (NSB-ACK) coding to reduce redundant packets.

Basically the candidate node relies on adding a hash vector to the packet header to disseminate its space information to other packets. When a relay overhears the hash from its neighbours, it can decide whether to produce linearly independent packets for them or to stop sending. CCACK utilizes an efficient credit based, rate control algorithm in addition to this acknowledgement scheme. CCACK increases both throughput and average of $45 \%$ and $8.8 \%$ respectively when compared with MORE.

The main limitation of these protocols is regarded through the use of a regular ETX measurement schemes based on probing which lead to incorrect values. As the actual relay node is chosen opportunistically, the routing method of these works is insensitive to ETX accuracy. The inaccuracy therefore only affects the candidate relay nodes in static wireless networks. In dynamic networks with high mobility, the issues becomes more prominent. Indeed, the probing technique in such networks no longer helpful as it does not provide any useful or accurate state information.

\subsection{PROBABILISTIC OPPORTUNISTIC ROUTING}

The purpose of probabilistic opportunistic routing protocols is to support mobility in wireless nodes effectively. This probabilistic approach is basicallyadopted by Burgess et al. in [15]. The authors proposed MaxProp, a joint scheduling and routing protocol. This protocol schedules the stored packets of the chosen node based on its cost being assigned. The packet delivery estimate is determined by the governing mobile node at each new encounter with another node in the network. Such mobile node holds a vector listing estimations of the required probability of meeting every other node.

The Fixed Point Opportunistic Routing (FPOR) protocol suggested by Conan et al[16] is used to enhance the general delay addressed by the average packet transmission. The authors deal with the design and analysis of two-hop relay setting and extend it recursively to the multihop environment. They also demonstrate that this routing strategy is at the fixed point of a recursive method and it provides maximum throughput. It is qualified as the probabilistic approach, because of its main procedure which is based exclusively on estimates of the average inter contact between pairs of the nodes in the network. These estimates were therefore obtained from distinct classical probability distributions of theintercontacttime interval like (Poisson,randomandexponential distributions) in order to make the issue tractable. It is also 
loop free and converges in polynomial time. Butthe real network dynamics is not taken intoconsideration. The development further encounter probabilities, rather than general, real system related entities.

Nelson et al. suggested EncounterBased Routing (EBR) [17] a quota-based protocol wherethe upper bound relay on the amount of replicas per mitted in the network that is stable during the creation of messages.For each node, EBR predicts an encounter value using past information and estimated value for forwarding the packets. The encounter value of each node depicts its rate of encounters and is determined using an exponentially weighted moving average technique. This protocol alsofocuses on the nodes that experience a large amount of encounters that are most likely to pass the message to the suitabledestination than the nodes that only infrequently encounter others. The nodes have higher priority are selected as the actual message forwarder node. The number of packet replicasis produced by the EBR protocolwhich is lower than the one caused by the Delegation Forwarding mechanism.

Pushpalatha et al. [18] proposed Disruption tolerant Secure Opportunistic Routing Protocol (DTSOR) for improving the network performance. In this method the routes are established based on the potential ability of the nodes and also trust worthy of the nodes in the neighbourhood are also considered for routing strategies. The node with the highest priority is considered as the best forwarder node for forwarding the packets. The Packet delivery ratio also increases when number of malicious node increases because of trust based evaluation procedure.

\subsection{CROSS LAYER OPPORTUNISTIC ROUTING}

Bletsas et al.[19 ] have suggested an opportunistic relay protocol that is best suited for noise and interference-limited slow fading environments. This protocol utilizes a distributed PHY-aware reactive relay selection scheme that requires no communication between relays.The bestrelay node selection is chosenbased on the link quality estimate towards the destination node. Each estimate of the relay node is owned by the Received Strength Signal Indicator (RSSI) which listens from the perspective of the location to a pilot signal.Based on the Carrier Sense Multiple Access method (CSMA), the candidate relay nodes are concerned with the medium, with a back off timer per relay being set depending on their own instantaneous signal strength. This protocol consists of two phases for implementing the relaying mechanism. In the first phase, the source node sends the data packet and in the second one, the selected best relay forwards this packet to the appropriate destination. The duration of these two phases is equal to the interval of coherence. Then it is assumed to be in the order of hundreds ofmilliseconds. Therefore without any delay the packetsarerelayed. The major restriction is that it can only be used to relay packets via two hops.

Lee et al. suggested a simple and practical Opportunistic Routing (SPOR) algorithm [20] for multi-hop wireless networks. SPOR comprises of iteratively forwarding a data packet and acknowledging its reception at each hop count. When the packet reaches the destination node, the latter will broadcast an ACK which will prevent furthertransmissionofthe 
packet.Theauthors also discussed the interference-aware assessment of the throughput performance of the proposed protocol. Theresultsshowthat the SPORachieveshigherthroughput and better performancethan traditionalrouting, which is used for short-haul paths. Theopportunistic route length is greater than four hops, and the performance gain becomes marginal. The limitation is that itconsiders only the linear one dimensional network model.

Mao et al.[21] proposed EEOR protocol, an energy-efficient opportunistic routing protocol for Wireless sensor networks. The protocol deals withthe selection of candidate relays and optimizes the priority system to minimize energy consumption. Moreover, the adjustable transmission power is also taken into consideration. The sender speeds up gradually its transmission energy up to a maximum limit, thus increasing the number of its neighbours. The sending nodereceives an increasing sequenceofCRS that corresponding to the level of transmission power levels. These CRSs are later sorted by their energetic costs. The major limitationof this protocol is that it does not take into account other parameters into considerationthat affects the energy consumption like interference.

Lu et al. suggested Position Based Opportunistic Routing PRO [22], an opportunisticrelaying-based link layer retransmission protocol for MANETs that uses the information provided by the PHY and the MAC layer for providing the successful packet retransmission. The PRO allows overhearing nodes to act as relays that retransmits the data to the destination nodes on behalf of the source after knowing the transmission of failure nodes. Toachieve the goal,using a run-time calibration process, the PROprotocol first estimates the instantaneous quality of the link to the destination. After using a local qualification process, it filters out the poor relays. Then the node with highest RSSI is chosen as the relay node for transmitting the packets to the destination. This protocol is compatible with conventional IEEE 802.11 standard and allows the co-existence of PROenabled and legacy IEEE 802.11 devices.

\subsection{OPTIMIZATION BASED OPPORTUNSTIC ROUTING}

Li et al. suggested a scalable opportunistic routing protocol, Localized Opportunistic Routing (LOR)[23].This protocolfirst utilizes a graph-theoretical scheme to partition the topology of wireless network into several comparatively small sub-topologies, called the Close Nodes Sets (CNSs). Based on the local information these CNSs are nested and constructed. The inter CNS and intra CNS routing is carried out by using a distributed Bellman-Fordalgorithm depending on whether or not the source and destination nodes lie within the same CNS. The LOR performs locally optimal opportunistic routing suitable for wireless large scale networks with lower complexity compared to MABF [24]. This protocol has a time complexity of $\mathrm{O}\left(\mathrm{n}^{2}\right)$, where $\mathrm{n}$ is the number of nodes in a CNS. This latter is much lower than the number of nodes in the whole network. The LOR achieves a trade-off between the global optimality of the used forwarder lists from its neighbourhood and routing scalability.

Fang et al. [25] addressed, the issue of opportunistic routing protocol subject to $\mathrm{K}$ constraints like time constraints, energy constraints, etc., The problem of NP-hardnessis achieved with polynomial time K-approximation when $\mathrm{K}>1$.Here, the Multi-constrained Any 
Path routing algorithm (MAP) based on a generalization of the SMAF algorithm [26] is used for solving it. This algorithm is very simple and is similar to Dijkstra's algorithm and each link in the network is assigned $\mathrm{K}$ weights related to $\mathrm{K}$ distinct parameters. These parameters are addressed by the $\mathrm{K}$ routing inequality constraints. The algorithm governs each node which is added to the candidate relay set thatdecreases the auxiliary weight of the corresponding hyperlink. The resulting opportunistic routing algorithm, MAP, corresponds to polynomial time complexity and provides a K-approximation of the optimal solution.

Xiao et al. [27] suggested Time-sensitive Opportunistic Utility-based Routing protocol (TOUR) for Delay Tolerant Networks (DTNs). This protocol utilizes an opportunistic routing algorithm that delivers data packets through short-delay yet costly paths. The packets are assigned with initial values that delay with time. The main aim of thisprotocol is to maximize the remaining time values of the messages when they reach their appropriate destinations. A continuous per message utility function is calculated from the difference of the transmission cost incurred by the packet delivery. Thetime-varying optimal forwarding method is used in order to address the problem of probabilistic nodes that encounters. Each node in the network forwards only messages to the encountered nodes in the timevarying sets. The messages are forwarded via nodes in these preselected sets, so TOUR achieves the optimal expected utilities. Table 1 shows the comparison of the routing protocols from the above categories.

Table 1 : Comparison of Opportunistic Routing Protocols

\begin{tabular}{|c|c|c|c|c|c|}
\hline rotocol & Category & $\begin{array}{l}\text { Coordination } \\
\text { Method }\end{array}$ & Performance Metric & Scheduling & Mobility \\
\hline POR & Geographic Routing & Timer & Geographical distance & $\begin{array}{l}\text { IEEE } \\
802.11\end{array}$ & Yes \\
\hline EXOR & $\begin{array}{l}\text { Link state Aware } \\
\text { Routing }\end{array}$ & $\begin{array}{l}\text { Timer and } \\
\text { Acknowledge } \\
\text { ment }\end{array}$ & $\begin{array}{l}\text { Expected Transmission } \\
\text { count(ETX) }\end{array}$ & Scheduled & No \\
\hline DTSOR & Probabilistic Routing & Overhearing & $\begin{array}{lr}\text { Packet } & \text { Delivery } \\
\text { Ratio(PDR) } & \text { and } \\
\text { Throughput } & \end{array}$ & Scheduled & Yes \\
\hline SPOR & $\begin{array}{ll}\text { Cross } & \text { layer } \\
\text { Opportunistic } & \\
\text { Routing } & \end{array}$ & $\begin{array}{l}\text { Acknowledgm } \\
\text { ent and } \\
\text { Overhearing }\end{array}$ & $\begin{array}{l}\text { Throughput and } \\
\text { Interference aware PDR }\end{array}$ & $\begin{array}{l}\text { RTS/CTS } \\
\text { based }\end{array}$ & No \\
\hline TOUR & Optimization based & Overhearing & $\begin{array}{l}\text { Packet Delivery ration } \\
\text { and Delay }\end{array}$ & No & Yes \\
\hline
\end{tabular}




\section{PERFORMANCE ANALYSIS OF OPPORTUNSTIC ROUTING PROTOCOLS}

The Network simulator -2 (NS-2) Simulator is used to analyse the performance of the Opportunistic Routing Protocols. The Simulation is carried with 100 nodes and the network transmission range is set as $250 \mathrm{~m}$. The nodes are uniformly distributed in the $1000 \mathrm{~m} \times 800 \mathrm{~m}$ rectangular region. The packet size of the node is 256 byes and Constant Bit Rate (CBR) is generated between the nodes. The simulation time is set at 500 seconds. The speed of a node is varied from $1 \mathrm{~m} / \mathrm{s}$ to $5 \mathrm{~m} / \mathrm{s}$ and mobility is introduced with Reference Point Group Mobility (RPGM) model.

Table 2 : PDR Comparison of Opportunistic Routing Protocols

\begin{tabular}{|l|l|l|l|l|l|}
\hline $\begin{array}{l}\text { Number of } \\
\text { nodes }\end{array}$ & POR & EXOR & DTSOR & SPOR & TOUR \\
\hline 20 & $81.4 \%$ & $62 \%$ & $82.8 \%$ & $79.8 \%$ & $79 \%$ \\
\hline 40 & $84.5 \%$ & 68 & $89 \%$ & $82.6 \%$ & $76 \%$ \\
\hline 60 & $83.4 \%$ & $70.2 \%$ & $92 \%$ & $80.1 \%$ & $78 \%$ \\
\hline 80 & $86.5 \%$ & $72 \%$ & $93.8 \%$ & $85 \%$ & $83 \%$ \\
\hline 100 & $85.8 \%$ & $71.8 \%$ & $95.7 \%$ & $84 \%$ & $79 \%$ \\
\hline
\end{tabular}

The Performance of the routing protocols are analysed based on Packet Delivery Ratio (PDR) and shown in Table 2. PDR is defined as a ratio of number of packets received by destination to number of packet sent by source. The simulation is carried out based on the node density. The Figure 2 shows the comparison of Packet DeliveryRatio (PDR) of the opportunistic protocols in highly mobility environment.As the size of the network increases, the distance betweennodes also increases and the probability of packet loss for all protocols also increases. Therefore, only fewer packets will get a chance to successfully reach the destination. A comparison of various protocols shows that DTSOR algorithm acts as the best algorithm for delivering the packets to the destination, whereas EXOR algorithm shows the worst delivery ratio with malicious nodes.As in case of DTSOR, the destination node receivesalmost all packets send by source. The packet delivery ratio of DTSOR is ranging from $97.02 \%$ to $99.43 \%$. The packet delivery ratio of other protocols ranging from $79.651 \%-91$. $75 \%$. Hence DTSOR chooses the route effectively even with high mobility and having better packet delivery ratio as compared to other protocols. The EXOR protocol uses Expected Transmission Count as the performance metric for choosing the candidate list and also for selecting the Forwarder list. DTSOR ensures the security of the nodes since it uses the trust based forwarding mechanism for forwarding the packets. In DTSOR, the cooperative nodes are identified based on the trust hence the malicious nodes are easily identified. 


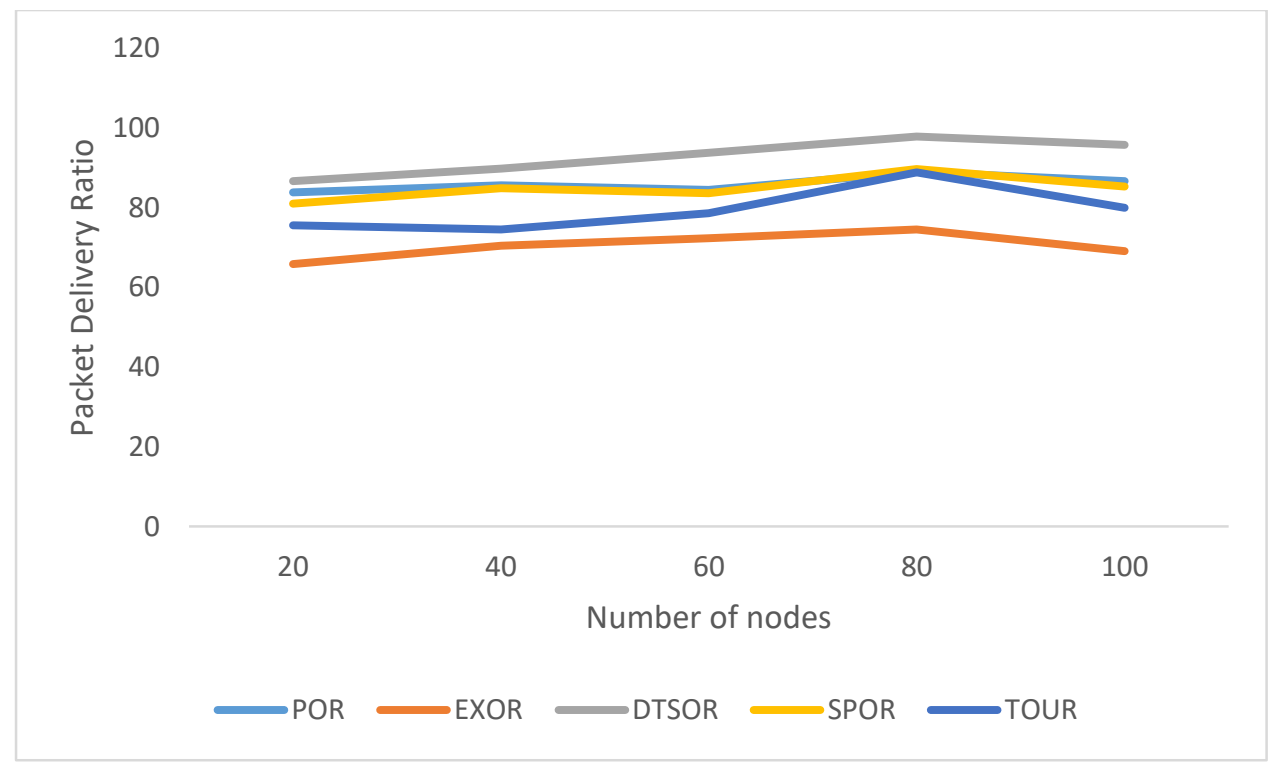

Fig 2. Performance comparison of Opportunistic Routing Protocols

Interestingly, however, POR almost outperforms both SPOR and EXOR.Because the POR protocol decreases the number of hops between source and destinations. This results in a lower probability of receiving packets to malicious nodes compared to SPOR and EXOR resulting in high PDR. TOUR still outperforms EXOR by integratingboth the link delivery probability between nodes, and their geographical information for candidate selection. The experimental results shows that the opportunistic approaches using probability provides better performance in highly dynamic wireless ad hoc networks.

\section{CONCLUSION}

A comprehensive survey of opportunistic routing protocols is discussed. The node location information is necessary for real time scenarios that includes gas leakage monitoring, and rescue operations. But, in terms of delay and reliability, these protocols may not be suited. Therefore, probabilistic opportunistic routing protocols have been taken into consideration that opts for other parameters, like link quality, weight, bandwidth and trust of a node. On comparison with the other categories of opportunistic routing, it is suitable for dynamic wireless networks, since it deals with the high mobility.Simulation results showed the behaviour and performance differences of these opportunistic routing protocols. The performance analysis clearly showed that DTSOR provides the better throughput and Packet Delivery Ratio when compared to other protocols in high mobility environments. It also ensures the security between the nodes in the network. This analysis would further help in designingadditional improvements in optimized opportunistic protocols forpreventing the invalid route that would guarantee very high quality of service in highly dynamic ad hoc networks. 


\section{REFERENCES}

1. Dhar, Subhankar. (2005). MANET: Applications, Issues, and Challenges for the Future. IJBDCN. 1. 66-92. 10.4018/jbdcn.2005040104.

2. Broch, J., Johnson, D., \& Maltz, D. (1999). The dynamic source routing protocol for mobile ad hoc networks(DSR). IETF, MANET Working Group. Internet draft 03

3. Haas, Z., \& Pearlman, M. (2000). The zone routing protocol (ZRP) for ad hoc network. IETF, MANET Working Group. Internet draft 03.

4. Perkins, Royer and Das. Ad hoc On-demand Distance Vector (AODV) Routing, RFC 3561. 2003 July; p. 1-37.

5. Aggarwal Akshai, Gandhi Savita, Chaubey Nirbhay. Performance analysis of AODV, DSDV and DSR in manets. International Journal of Distributed and Parallel Systems (IJDPS). 2011 November; 2(6):11.

6. A. Martin-Campillo, J. Crowcroft, E. Yoneki, and R. Marti, "Evaluatingopportunistic networks in disaster scenarios," J. Netw. Comput. Appl.,vol. 36, no. 2, pp. 870-880,2013

7. H. Liu, B. Zhang, H. Mouftah, X. Shen, and J. Ma, "Opportunistic routing for wireless ad hoc and sensor networks: Present and future directions," IEEE Commun. Mag., vol. 47, no. 12, pp. 103-109,Dec. 2009.

8. H. Fussler, J. Widmer, M. Kasemann, M. Mauve, and H. Hartenstein, "Contention-based forwarding for mobile ad-hoc networks," Ad HocNetw., vol. 1, no. 4, pp. 351-369, Nov. 2003.

9. K. Zeng,W. Lou, J. Yang, and D. Brown III, “On throughput efficiency of geographic opportunistic routing in multihop wireless networks," MobileNetw. Appl., vol. 12, no. 5, pp. 347-357, Dec. 2007.

10. S. Yang, F. Zhong, C. Yeo, B. Lee, and J. Boleng, "Position based opportunistic routing for robust data delivery in MANETs," in Proc.IEEE Conf. GLOBECOM, Dec. 2009, pp. 1-6.

11. S. Biswas and R. Morris, "Opportunistic routing in multi-hop wirelessnetworks," in Proc. Conf. ACM SIGCOMM, Aug. 2005, pp. 69-74.

12. C. Hsu, H. Liu, and W. Seah, "Economy: A duplicate free opportunistic routing," in Proc. 6th ACM In. Conf. Mobile Technol. Appl. Syst., 2009,pp. 17:1-17:6.

13. S. Chachulski, M. Jennings, S. Katti, and D. Katabi, "Trading structure for randomness in wireless opportunistic routing," in Proc. Conf. ACMSIGCOMM, Oct. 2007, pp. 169-180.

14. D. Koutsonikolas, C. Wang, and Y. Hu, "CCACK: Efficient network coding based opportunistic routing through cumulative coded acknowledgments,"in Proc. IEEE Conf. INFOCOM, 2010, pp. 1-9.

15. J. Burgess, B. Gallagher, D. Jensen, and B. N. Levine, "MaxProp: Routing for vehicle-based disruption-tolerant networks," in Proc. 25th IEEEConf. INFOCOM, Apr. 2006, pp. 1-11.

16. J. L. V. Conan and T. Friedman, "Fixed point opportunistic routing in delay tolerant networks," IEEE J. Sel. Areas Commun., vol. 26, no. 5,pp. 773-782, Jun. 2008.

17. S. Nelson, M. Bakht, and R. Kravets, "Encounter-based routing in DTNs," in Proc. IEEE INFOCOM, Apr. 2009, pp. 846-854.

18. K. Pushpalatha, M. Karthikeyan, "A generalized framework for disruption tolerant secure opportunistic routing during emergency situations using MANETs" in Cluster Computing, New York, NY, USA:Springer, pp. 1-9, 2018.

19. A. Bletsas, A. Dimitriou, and J. Sahalos, "Interference-limited opportunistic relaying with reactive sensing," IEEE Trans. Wireless Commun.,vol. 9, no. 1, pp. 14-20, Jan. 2010. 
20. G. Lee and Z. Haas, "Simple, practical, effective opportunistic routing for short-haul multi-hop wireless networks," IEEE Trans. WirelessCommun., vol. 10, no. 11, pp. 3583-3588, Nov. 2011.

21. X. Mao, S. Tang, X. Xu, X. Li, and H. Ma, "Energy efficient opportunistic routing in wireless sensor networks," IEEE Trans. Parallel Distrib.Syst., vol. 22, no. 11, pp. 1934-1942, Nov. 2011.

22. M. Lu, P. Steenkiste, and T. Chen, "Design, implementation and evaluation of an efficient opportunistic retransmission protocol," in Proc.IEEE/ACM Annu. Int. Conf. MobiCom, Sep. 2009, pp. 73-84.

23. Y. Li, A. Mohaisen, and Z. Zhang, "Trading optimality for scalability in large-scale opportunistic routing," IEEE Trans. Veh. Technol., vol. 62,no. 5, pp. 2253-2263, Jun. 2013.

24. R. Laufer, H. Dubois-Ferriere, and L. Kleinrock, "Polynomial-time algorithms for multirate anypath routing in wireless multihop networks," IEEE/ACM Trans. Netw., vol. 20, no. 3, pp. 742-755, Jun. 2012.

25. X. Fang, D. Yang, and G. Xue, "MAP: Multi-constrained anypath routing in wireless mesh networks," IEEE Trans. Mobile Comput., vol. 12,no. 10, pp. 1893-1906, Oct. 2013.

26. R. Laufer, H. Dubois-Ferriere, and L. Kleinrock, "Multirate anypath routing in wireless mesh networks," in Proc. IEEE Conf. INFOCOM,2009, pp. 37-45.

27. M. Xiao, J. Wu, K. Liu, and L. Huang, "TOUR: Time-sensitive opportunistic utility-based routing in delay tolerant networks," in Proc. IEEEConf. INFOCOM, 2013, pp. 2085-2091. 